\title{
Non-ideal monitoring of a qubit state using a quantum tunnelling device
}

\author{
Neil Oxtoby $\dagger+$, He-Bi Sun $\uparrow \S$ and Howard M Wiseman $\ddagger$ \\ $\dagger$ Centre for Quantum Computer Technology, Department of Physics, University of \\ Queensland, St. Lucia, QLD 4072, Australia \\ $\ddagger$ Centre for Quantum Computer Technology, School of Science, Griffith University, \\ Nathan, QLD 4111, Australia
}

\begin{abstract}
We propose a model for non-ideal monitoring of the state of a coupled quantum dot qubit by a quantum tunnelling device. The non-ideality is modelled using an equivalent measurement circuit. This allows realistically available measurement results to be related to the state of the quantum system (qubit). We present a quantum trajectory that describes the stochastic evolution of the qubit state conditioned by tunnelling events (i.e. current) through the device. We calculate and compare the noise power spectra of the current in an ideal and a non-ideal measurement. The results show that when the two qubit dots are strongly coupled the non-ideal measurement cannot detect the qubit state precisely. The limitation of the ideal model for describing a realistic system may be estimated from the noise spectra.
\end{abstract}

PACS numbers: 85.35. Gv,85.35.Gv, 03.65.Ta, 42.50.Lc, 03.67.Lx

Submitted to: J. Phys.: Condens. Matter

\section{Introduction}

For a quantum computer to be practical, one of the important questions is how to readout the final results of the quantum computation reliably. Measurement of the state of qubits, the two-state systems, at a single-electron level is essential for a solid-state quantum computer [1]. Most proposals for the measurement of quantum systems are idealized $[2,3,4]$. However, in a real laboratory a perfect measurement is hardly possible due to practical devices and circuitry. We try to model imperfect measurements so that realistically available results can be related to the state of the quantum system (in this case, a solid state qubit). Quantum point contacts (QPCs) [5, 6] and single electron transistors (SETs) $[2,3,7]$ are popular quantum tunnelling devices in proposals for measurement of coupled-dot systems. Here we study continuous monitoring of the state of a pair of coupled quantum dots by a quantum tunnelling (QT) device. We include $\S$ To whom correspondence should be addressed (sun@physics.uq.edu.au) 
the case of imperfect (non-ideal) measurement. The pair of dots, occupied by a single electron tunnelling coherently between them, act as a qubit $[8,9]$.

Fluctuations in time of a measurement can be a source of information that may be difficult or impossible to be directly probed by measurement of time-averaged quantities. Current fluctuations due to the discreteness of the electrical charge play a diagnostic role similar to that in photon measurements. However, the correlations between electrons due to the Pauli principle introduce extra features of quantum noise in mesoscopic systems based on the the system states and reflected in the noise spectrum [10]. In this study we use the current noise spectrum to obtain information about the quantum processes within the coupled dots.

The paper is organized as follows. The details of the system are described in Section 2. The formalism is presented in Section 3, where we present a quantum trajectory that describes the stochastic evolution of the qubit state when measured by a QT device. In Section 4 we show and analyze the calculated results and compare the noise spectra of measured current for the cases of ideal and non-ideal measurement. We summarize our results in Section 5. We found that noise as an informative signal in mesoscopic systems indeed provides information about the qubit state and a non-ideal measurement fails to obtain information about the quantum processes occurring within the qubit when the two dots are strongly coupled. The limitations of the modelling with an ideal device, estimated from the noise power spectra, are discussed.

\section{Description of the measurement and system}

The quantum system to be measured is a pair of spatially separated and coherently coupled quantum dots occupied by a single electron. Each dot is assumed to have only one available state. The interaction between the QT device and the nearer dot is via a Coulomb interaction. The state of the qubit at a particular time is described by the location of the confined electron at that time. The electron tunnelling rate through the measurement device is affected by the location of the qubit electron: when the electron occupies the further dot-2, the rate is denoted by $\lambda_{0}$ while an additional rate $\lambda_{1}(>0)$ occurs when the electron is in the nearer dot-1. Hence the QT device operates as a measurement device to detect the state of the qubit. The quiescent tunnelling rate $\lambda_{0}$ is usually nonzero due to Johnson-Nyquist noise and other factors such as defects in the device.

This model is based on that of reference [2] which considered a single electron transistor (SET) with adiabatically eliminated island dot. When the quiescient rate of tunnelling through the device is negligible $\left(\lambda_{0} \ll \lambda_{1}\right)$, this model is equivalent to that of a low transparency quantum point contact [6].

In the case of ideal measurement, the current through the QT device involves only tunnelling events that reflect the qubit's state. In this case, the only noise present is the quantum noise due to the stochastic nature of the tunnelling processes through the QT device. However, for non-ideal measurement of the qubit state, the measured 


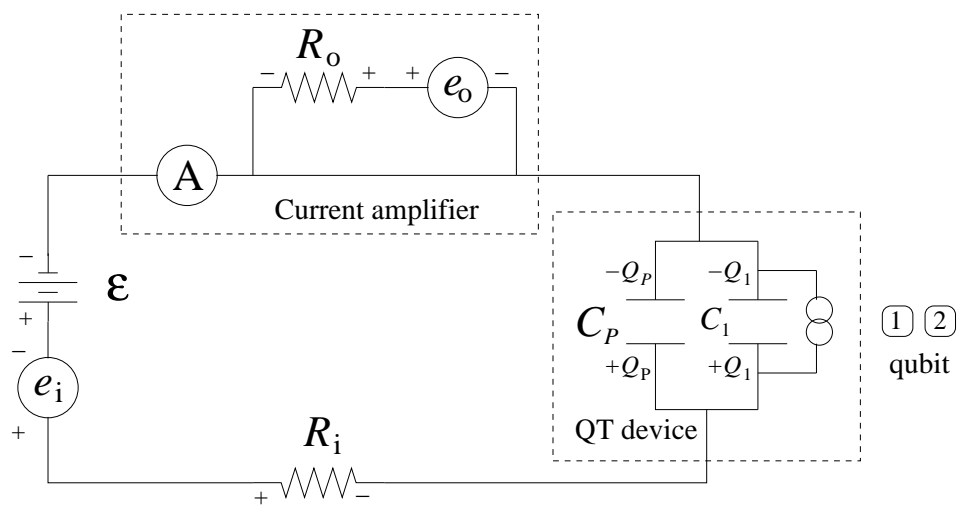

Figure 1. Equivalent circuit for measurement of current through the QT device. The single tunnel junction in the QT device is modelled as a capacitor $C_{1}$. A parasitic capacitance, $C_{\mathrm{P}}$, between the source and drain is included in parallel with the QT device. Tunnelling events through the device are modelled as a current source.

current includes extra noise components. The extra noise is caused by classical noise sources from the real measurement components. We use an equivalent circuit to model the realistic measurement as shown in figure 1. The circuit is structured in three parts: the QT device, a current amplifier and miscellaneous circuit components. The QT device tunnel junction is represented by a capacitance, $C_{1}$, in parallel with a parasitic capacitance, $C_{\mathrm{P}}$, that exists between the source and drain 2DEGs. The parasitic capacitance $C_{\mathrm{P}}$ is generally larger than the junction capacitance $C_{1}$ due to its larger 'area'. In the formalism, we consider the equivalent parallel capacitance $C=C_{1}+C_{\mathrm{P}}$. The DC bias voltage consists of an ideal electromotive force, $\varepsilon$, in series with an input noise voltage source $e_{\mathrm{i}}$, which includes the Johnson-Nyquist noise of the equivalent resistance $R_{\mathrm{i}}$. (These circuit components introduce an input noise into the current through the QT device). The current through the QPC is amplified by a non-ideal current amplifier. This is modelled as an ammeter that contributes an output noise $e_{\mathrm{o}} / R_{\mathrm{o}}$ into the measured current $I(t)$, where $R_{\mathrm{o}}$ is the resistance associated with the non-ideal amplifier, which is at temperature $T_{\mathrm{o}} \sim 4 K$. Tunnelling events through the QT device are modelled as a current source. The detailed description of the measured current at time $\mathrm{t}, \mathrm{I}(\mathrm{t})$, including effects of the realistic components is expressed in the second part of the following section.

\section{Theoretical modelling and stochastic approach}

First we present the formalism in the ideal measurement case. The total Hamiltonian of the qubit dots can be expressed as

$$
H=\hbar \sum_{j=1}^{2} \omega_{j} c_{j}^{\dagger} c_{j}+i \hbar \frac{\Omega}{2}\left(c_{1}^{\dagger} c_{2}-c_{2}^{\dagger} c_{1}\right),
$$


where $\Omega$ is the coupling frequency between two dots and $c_{j}$ and $c_{j}^{\dagger}(j=1,2)$ are the annihilation and creation operators for the single electron states within the qubit dots. The first and second terms of the right hand side of equation (1) are the quasi-boundstate energies and the interaction between the two dots, respectively. The average dynamics of the qubit are described by the following unconditional quantum master equation $[2]$

$$
\begin{aligned}
\frac{\mathrm{d} \rho(t)}{\mathrm{d} t} & =-i[H, \rho(t)]+\gamma \mathcal{D}\left[n_{1}\right] \rho(t) \\
& \equiv \mathcal{L} \rho(t),
\end{aligned}
$$

where $\gamma=2 \lambda_{0}+\lambda_{1}$ is the decoherence rate of the qubit [2], $\lambda_{0}$ and $\lambda_{1}$ are the tunnelling rates introduced in the previous section, $n_{1}=c_{1}^{\dagger} c_{1}$ is the occupation number of dot- 1 and $\mathcal{L}$ is the Liouvillian super-operator. The operator $\mathcal{D}[X] Y$ is defined by

$$
\begin{aligned}
\mathcal{D}[X] Y & \equiv \mathcal{J}[X] Y-\mathcal{A}[X] Y \\
& =X Y X^{\dagger}-\frac{1}{2}\left(X^{\dagger} X Y+Y X^{\dagger} X\right) .
\end{aligned}
$$

Note that the convention of $\hbar=1$ is chosen here. The master equation, being of the Lindblad form [11] for valid evolution (that is, preserving the Hermiticity, norm and positivity of $\rho$ ), was derived in the appendix of reference [2] for a SET with adiabatically eliminated island dot. In the limit of $\lambda_{0} \ll \lambda_{1}$, the model is equivalent to that of a low transparency QPC in reference [6]. In both cases, the QT device considered has a single junction through which electrons must tunnel.

We define the ideal current through the QT device in terms of the discrete Poissonian process $d N(t)$ :

$$
i(t)=q \frac{\mathrm{d} N(t)}{\mathrm{d} t},
$$

where $q=-|q|$ is the charge on an electron. The classical point process $d N(t)$ is defined by the conditions:

$$
\begin{aligned}
\mathrm{d} N(t)^{2} & =\mathrm{d} N(t) \\
\mathrm{E}\left[\frac{\mathrm{d} N(t)}{\mathrm{d} t}\right] & =\lambda_{0} \operatorname{Tr}\left[\left(1-n_{1}\right) \rho_{\mathrm{c}}(t)\left(1-n_{1}\right)\right]+\left(\lambda_{0}+\lambda_{1}\right) \operatorname{Tr}\left[n_{1} \rho_{\mathrm{c}}(t) n_{1}\right] \\
& =\lambda_{0}+\lambda_{1}\left\langle n_{1}\right\rangle_{\mathrm{c}}(t) .
\end{aligned}
$$

Notice that the (classical) expectation value has been expressed as a quantum average. The conditions indicate that $\mathrm{d} N(t)$ equals zero or one and that the rate of the tunnelling events through the QT device is equal to the background rate plus an additional rate $\lambda_{1}$ if and only if the electron is in dot-1.

The quantum trajectory (stochastic master equation) for the case of ideal measurement is $[2]$

$$
\begin{aligned}
\mathrm{d} \rho_{\mathrm{c}}= & \mathrm{d} N\left[\frac{\mathbf{J}}{\operatorname{Tr}\left[\mathbf{J} \rho_{\mathrm{c}}\right]}-1\right] \rho_{\mathrm{c}} \\
& +\mathrm{d} t\left\{-\lambda_{1} \mathcal{A}\left[n_{1}\right] \rho_{\mathrm{c}}+\lambda_{1} \operatorname{Tr}\left[\rho_{\mathrm{c}} n_{1}\right] \rho_{\mathrm{c}}-i\left[H, \rho_{\mathrm{c}}\right]\right\}
\end{aligned}
$$


where the super-operator $\mathbf{J}$ is defined as $\mathbf{J} \rho_{\mathrm{c}} \equiv \lambda_{0} \rho_{\mathrm{c}}+\lambda_{1} \mathcal{J}\left[n_{1}\right] \rho_{\mathrm{c}}+2 \lambda_{0} \mathcal{D}\left[n_{1}\right] \rho_{\mathrm{c}}$, the superoperators $\mathcal{J}$ and $\mathcal{A}$ were defined implicitly by equation (3) and the time argument is omitted for simplicity. The expectation value of $\mathrm{d} N(t)$ can therefore be expressed in terms of $\mathbf{J}$ as

$$
\mathrm{E}\left[\frac{\mathrm{d} N(t)}{\mathrm{d} t}\right]=\operatorname{Tr}\left[\mathbf{J} \rho_{\mathrm{c}}(t)\right] .
$$

The subscript $\mathrm{c}$ indicates that the stochastic evolution of the state matrix is conditioned on tunnelling events through the QT device at earlier times. Averaging the quantum trajectory over the observed stochastic processes recovers the unconditional master equation, (2).

Using Kirchhoff's laws to analyze the equivalent circuit of figure 1, we obtain the Itô differential equation [12] for the charge on the parasitic capacitor, $Q(t)$, and the expression for the (non-ideal) measured current.

The Itô differential equation for $Q(t)$ is

$$
\mathrm{d} Q(t)=[-\alpha Q(t)+\beta] \mathrm{d} t+\sqrt{D_{\mathrm{i}}} \mathrm{d} W_{\mathrm{i}}(t)+q \mathrm{~d} N(t),
$$

where $\mathrm{d} W_{\mathrm{i}}(t)$ is the input noise Wiener process [12], $\alpha=1 / R_{\mathrm{i}} C, \beta=\varepsilon / R_{\mathrm{i}}, D_{\mathrm{i}}=$ $2 k_{\mathrm{B}} T_{\mathrm{i}} / R_{\mathrm{i}}, T_{\mathrm{i}}$ is the laboratory temperature and $k_{\mathrm{B}}$ is Boltzmann's constant. The positive sign on the tunnelling increment is due to our definition of the direction of the current in the circuit.

Our circuit analysis yielded the following expression for the measured current as a function of time

$$
I(t)=-\alpha Q(t)+\beta+\sqrt{D_{\mathrm{i}}} \frac{\mathrm{d} W_{\mathrm{i}}(t)}{\mathrm{d} t}+\sqrt{D_{\mathrm{o}}} \frac{\mathrm{d} W_{\mathrm{o}}(t)}{\mathrm{d} t},
$$

where $D_{\mathrm{o}}=2 k_{\mathrm{B}} T_{\mathrm{o}} / R_{\mathrm{o}}, T_{\mathrm{o}}$ is the amplifier temperature and $\mathrm{d} W_{\mathrm{o}}(t)$ is the output noise Wiener increment due to the amplifier.

It is straight-forward to find the solution of equation (9) as

$$
Q(t)=\frac{\beta}{\alpha}+\sqrt{D_{\mathrm{i}}} \mathrm{e}^{-\alpha t} \int_{-\infty}^{t} \mathrm{e}^{\alpha t_{1}} \frac{\mathrm{d} W_{\mathrm{i}}\left(t_{1}\right)}{\mathrm{d} t_{1}} \mathrm{~d} t_{1}+q \mathrm{e}^{-\alpha t} \int_{-\infty}^{t} \mathrm{e}^{\alpha t_{1}} \frac{\mathrm{d} N\left(t_{1}\right)}{\mathrm{d} t_{1}} \mathrm{~d} t_{1} .
$$

The current is therefore given by substitution of equation (11) into equation (10):

$$
\begin{aligned}
I(t)= & -\alpha \sqrt{D_{\mathrm{i}}} \mathrm{e}^{-\alpha t} \int_{-\infty}^{t} \mathrm{e}^{\alpha t_{1}} \frac{\mathrm{d} W_{\mathrm{i}}\left(t_{1}\right)}{\mathrm{d} t_{1}} \mathrm{~d} t_{1}-\alpha q \mathrm{e}^{-\alpha t} \int_{-\infty}^{t} \mathrm{e}^{\alpha t_{1}} \frac{\mathrm{d} N\left(t_{1}\right)}{\mathrm{d} t_{1}} \mathrm{~d} t_{1} \\
& +\sqrt{D_{\mathrm{i}}} \frac{\mathrm{d} W_{\mathrm{i}}(t)}{\mathrm{d} t}+\sqrt{D_{\mathrm{o}}} \frac{\mathrm{d} W_{\mathrm{o}}(t)}{\mathrm{d} t} .
\end{aligned}
$$

One may argue that the current $I(t)$, rather than the point process $\mathrm{d} N / \mathrm{d} t$, is measured in a real experiment. It is indeed that the realistic conditional state of the system would be conditioned upon $I(t)$. This can be realized by following the method introduced for photodetectors in references [13] and [14]. The result is a stochastic Fokker-Planck equation for $\rho_{\mathrm{c}}(Q)$, where $\operatorname{Tr} \rho_{\mathrm{c}}(Q)$ is the conditional probability that the charge on the capacitor is $Q$, and $\int \mathrm{d} Q \rho_{\mathrm{c}}(Q)$ is the conditional quantum state, averaged over the unobserved charge $Q$. The details of this equation and its derivation will be presented elsewhere. 
In case it is not obvious, we use $i(t)$ to denote an ideal current (consisting only of tunnelling events through the detector) and $I(t)$ to denote a non-ideal current that contains extra noises introduced by the realistic measurement circuit.

\section{Current noise spectra}

For the noise involved in the detection of qubit states by a QT device, two types of noise are considered in this study: Johnson-Nyquist noise due to thermal motion of electrons that does not provide quantum information and shot noise due to the discreteness of the charge of electrons. In the steady state as well as many practical situations, when electron pulse widths are less than $1 / \omega$, Johnson noise is white noise which has a flat power spectrum. The current noise spectrum is given by $S_{\text {Johnson }}=4 k_{\mathrm{B}} T / R$, where $T$ is the absolute temperature of the conductor and $R$ is the conductor resistance. This noise therefore provides only the temperature value and no information about the quantum states.

In (single) tunnel junction devices the transfer of electrons can be described by Poisson statistics and the shot noise has its maximum value $S_{\text {shot }}=2 q I_{\mathrm{m}} \equiv S_{\text {Poisson }}$, where $I_{\mathrm{m}}$ is the time-averaged mean current through the device. The shot noise can be suppressed below $S_{\text {Poisson }}$ by correlations due to the Pauli exclusion principle and is a source of information of the quantum system involved in the measurement $[10,15]$. Noise is characterized by its power-density spectrum $S(\omega)$, which is the Fourier transform of the current-current two-time autocorrelation function [16], $G(\tau)$ :

$$
G(\tau)=\langle I(t) I(t+\tau)\rangle_{\mathrm{ss}}-\langle I(t)\rangle_{\mathrm{ss}}\langle I(t+\tau)\rangle_{\mathrm{ss}},
$$

where $I(t)$ represents the current through the QPC as a function of time and the subscript ss denotes the steady-state. The noise spectrum is expressible as [17]

$$
S(\omega)=4 \int_{0}^{\infty} G(\tau) \cos (\omega \tau) \mathrm{d} \tau
$$

We use dimensionless parameters, normalizing $S(\omega)$ by the full shot noise level $2 q I_{\mathrm{ss}}$ to produce what is known as the Fano factor [18]

$$
F(\omega)=\frac{S(\omega)}{2 q I_{\mathrm{ss}}}
$$

where the time averaged mean current in this case is the steady-state current $I_{\mathrm{ss}}$.

In the ideal measurement case the noise is purely due to the stochastic nature of the quantum processes. The current tunnelling through the QT device in this case is described by equation (4) and the following steady-state autocorrelation function can be obtained using the definition (13)

$$
G(\tau)=q i_{\mathrm{ss}} \delta(\tau)+\frac{\mathrm{e}^{2} \lambda_{1}^{2}}{8}\left(\frac{b_{+} \mathrm{e}^{b_{-} \tau}-b_{-} \mathrm{e}^{b_{+}}}{\sqrt{(\gamma / 4)^{2}-\Omega^{2}}}\right)
$$

where

$$
b_{ \pm}=-\gamma / 4 \pm \sqrt{(\gamma / 4)^{2}-\Omega^{2}}
$$



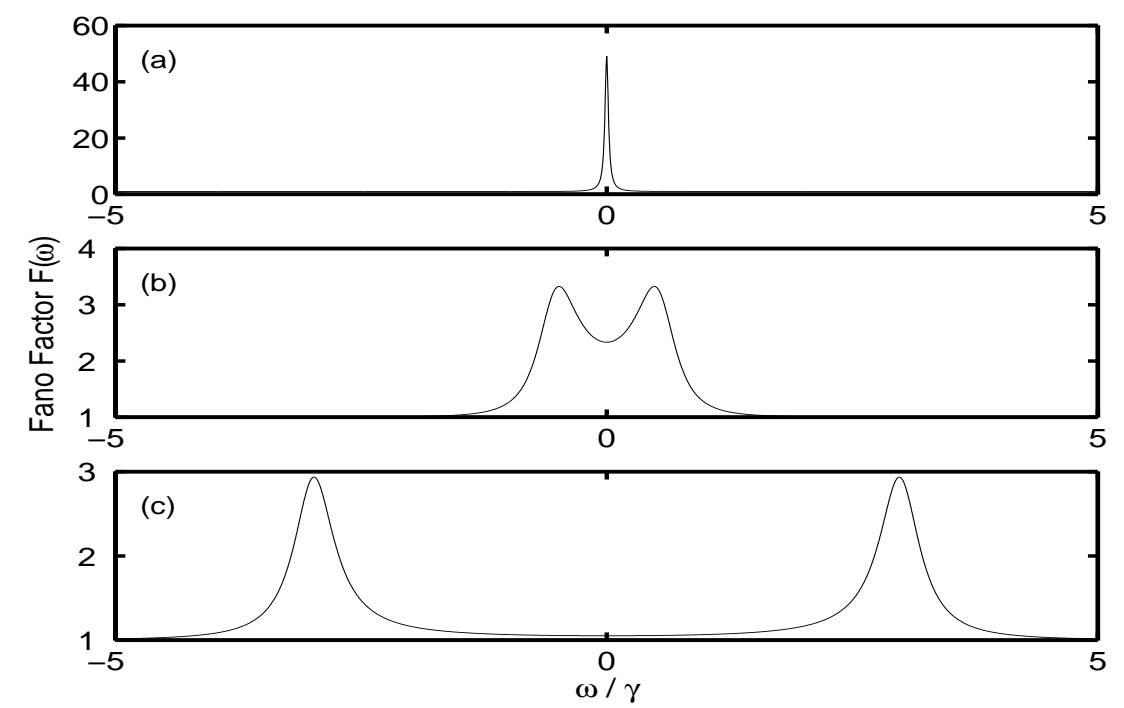

Figure 2. Fano Factor plots for ideal measurement of the current for different values of the tunnel coupling between qubit dots: (a) $\Omega=0.1 \gamma$, (b) $\Omega=0.6 \gamma$, (c) $\Omega=3 \gamma$.

are two (possibly complex) numbers. The Fourier transform of $G(\tau)$ gives the noise spectrum:

$$
S(\omega)=2 q i_{\mathrm{ss}}+\frac{\mathrm{e}^{2} \lambda_{1}^{2} \Omega^{2}}{2 \sqrt{(\gamma / 4)^{2}-\Omega^{2}}}\left[\frac{1}{b_{+}^{2}+\omega^{2}}-\frac{1}{b_{-}^{2}+\omega^{2}}\right] .
$$

We plot the noise spectra (as a Fano Factor plot) for the case of ideal measurement in figure 2 for three different values of $\Omega$ corresponding to the cases of (a) weak, (b) intermediate and (c) strong coupling between the two dots, respectively. The double peaked structure indicates coherent tunnelling between the qubit dots. The separation of the peaks is a measure of the strength of the tunnel coupling - larger separation corresponds to stronger coherence in tunnelling between the qubit dots $[2,15]$.

The measured current in the non-ideal circuit is more complicated, as shown in equation (10). The corresponding two-time correlation function and noise spectrum are calculated as the following equations:

$$
\begin{aligned}
G(\tau)= & q I_{\mathrm{ss}} \delta(\tau)+D_{\mathrm{o}} \delta(\tau)+D_{\mathrm{i}}\left(\delta(\tau)-\frac{\alpha}{2} \mathrm{e}^{-\alpha \tau}\right) \\
& +\frac{\alpha^{2} \mathrm{e}^{2} \lambda_{1}^{2}}{8 \sqrt{(\gamma / 4)^{2}-\Omega^{2}}}\left\{\frac{b_{+}}{\alpha^{2}-b_{-}^{2}} \mathrm{e}^{b_{-} \tau}-\frac{b_{-}}{\alpha^{2}-b_{+}^{2}} \mathrm{e}^{b_{+} \tau}\right. \\
& \left.+\left(\frac{b_{+}}{\alpha^{2}-b_{-}^{2}}-\frac{b_{-}}{\alpha^{2}-b_{+}^{2}}+\frac{b_{-}}{\alpha\left(\alpha+b_{+}\right)}-\frac{b_{+}}{\alpha\left(\alpha+b_{-}\right)}\right) \mathrm{e}^{-\alpha \tau}\right\}
\end{aligned}
$$




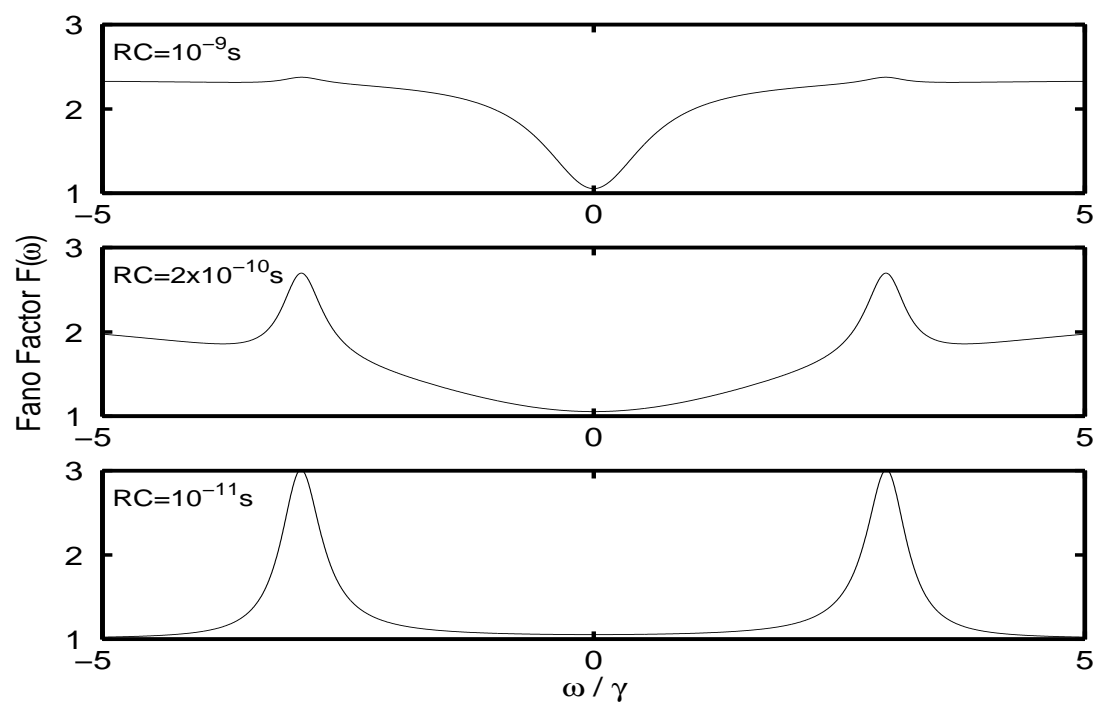

Figure 3. Fano Factor plot for non-ideal measurement of the current when the coupling between the qubit dots is relatively strong: $\Omega=3 \gamma$. The values of $R_{\mathrm{i}} C$ are given in the plots.

$$
\begin{aligned}
S(\omega)= & 2 q I_{\mathrm{ss}}+2 D_{\mathrm{o}}+2 D_{\mathrm{i}}\left(1-\frac{\alpha^{2}}{\alpha^{2}+\omega^{2}}\right) \\
& +\frac{\mathrm{e}^{2} \lambda_{1}^{2} \Omega^{2}}{2 \sqrt{(\gamma / 4)^{2}-\Omega^{2}}}\left[\frac{1}{b_{+}^{2}+\omega^{2}}-\frac{1}{b_{-}^{2}+\omega^{2}}\right]\left(\frac{\alpha^{2}}{\alpha^{2}+\omega^{2}}\right)
\end{aligned}
$$

Again for catching and comparing the corresponding quantum features, we visualize the characteristics by plotting the noise spectra in the non-ideal measurement case for various parameters. Figures 3, 4 and 5 correspond to strong, intermediate and weak coupling strength between the qubit dots, respectively. For comparison with the ideal case, the coupling strength $\Omega$ between the qubit dots in figures 3,4 and 5 are chosen as the same values for figure 2 (c), (b), and (a), respectively.

The influence of the non-ideal circuit components on the noise spectra is most significant in the strong coupling case as shown in figure 3 where $\Omega=3 \gamma$. From the top to the bottom, the parasitic parameters decrease by two orders. The top plot corresponds to the parasitic components of $R_{\mathrm{i}}=100$ Ohms and $C=10 \mathrm{pF}$, which are from the literature [19]. The sharp peaks in the noise spectrum of the ideal measurement are suppressed into small bumps here due to the imperfect measurement-circuit, and the original spectral features that provide qubit-state information are almost lost. As the parasitic capacitance is decreased (the lower two plots) in figure 3 , the original features of the ideal noise spectrum in figure 2 (c) are gradually recovered.

Figure 4 represents the intermediate tunnel coupling strength between the qubit $\operatorname{dots}(\Omega=0.6 \gamma)$. It shows a weaker influence of the non-ideal circuit components on the features of the noise spectrum. For comparison, the values of the parasitic components 


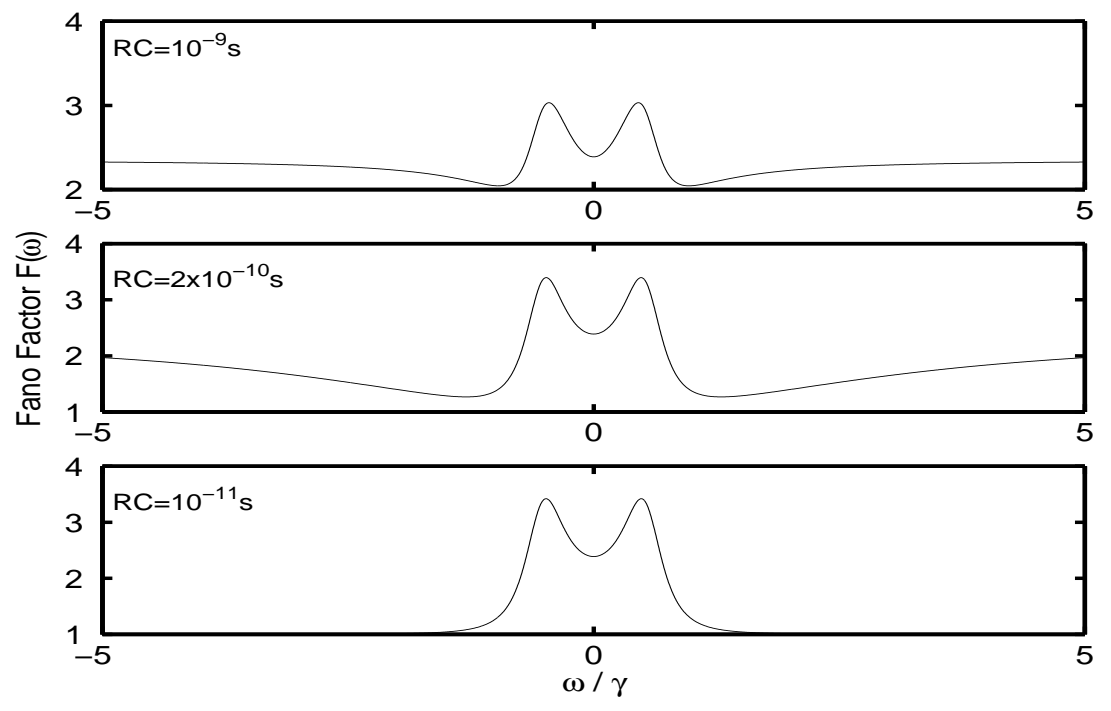

Figure 4. Fano Factor plot for non-ideal measurement of the current when the coupling rate between the qubit dots is an intermediate value: $\Omega=0.6 \gamma$. The values of $R_{\mathrm{i}} C$ are given in the plots.
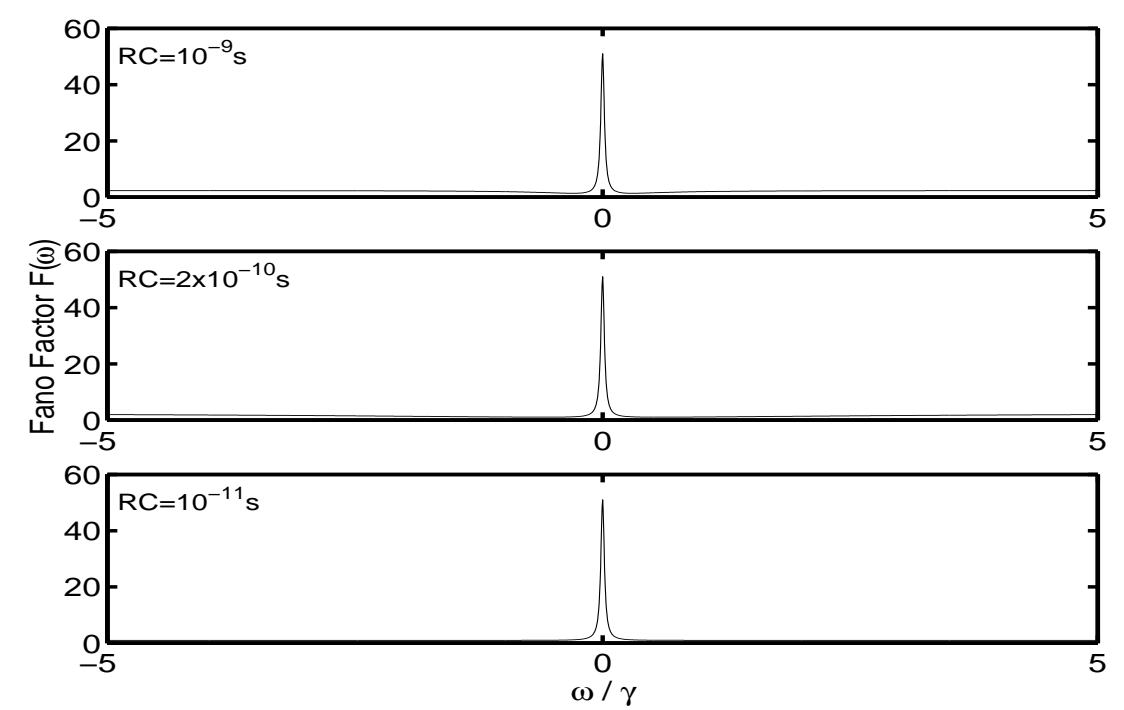

Figure 5. Fano Factor plots for non-ideal measurement of the current when the coupling between the qubit dots is weak: $\Omega=0.1 \gamma$. The values of $R_{\mathrm{i}} C$ are given in the plots.

are the same as in figure 3. The filter shape (the wings in the spectrum) remains identical, but the peaks are not suppressed by as much as for the stronger coupling case. The peaks showing the coupling strength between the qubit dots are easily visible for all three values of $R_{\mathrm{i}} C$. That is, for intermediate coupling strength between the qubit dots $(\Omega \gtrsim \gamma / 4)$, information about the qubit state can be obtained by non-ideal measurement (provided $R_{\mathrm{i}} C<10^{-9} s$ ).

The noise spectra for weak coupling between the qubit dots, shown in figure 5 , 
are very close to the spectrum in the ideal case in figure 2 (a). So, for weak inter-dot coupling, the non-ideal circuit components have a negligible influence on the qubit-state information that is written in the features of the measured current noise spectrum.

We draw the conclusion that the noise spectrum therefore acts as a diagnostic tool that can be used to estimate whether a measurement device of known parameters can be modelled as ideal or if the dynamics of the quantum system can be detected by such a device/circuit.

\section{Summary}

We have analyzed the measurement of the dynamics of a coupled quantum dot (qubit) system by a quantum tunnelling (QT) device using the quantum stochastic approach. This approach describes the evolution of the qubit state conditioned on a particular realisation of current through the detector in the form of a quantum trajectory (stochastic master equation). We have presented results for both an ideal and a non-ideal measurement using a low transparency QPC. The non-ideal measurement was modelled by an equivalent circuit. We pointed out that the current noise power spectrum can be used as a diagnostic tool to detect information about the qubit dynamics and the influence of the parasitic (circuit) components. We found that, in general, the non-ideal circuit components increased the current noise. The influence of the non-ideal circuit components on the features of the current noise spectrum that provide information about the qubit (i.e. the peaks) is greatest for the case of strong coupling between the qubit dots, when it is difficult to obtain information about the quantum processes within the qubit in a non-ideal measurement. We concluded that the current noise spectrum may be used to determine the limits of applicability of the ideal model to a realistic measurement.

\section{References}

[1] Kane B E 1998 Nature 393133

[2] Wiseman H M et al 2001 Phys. Rev. B 63235308

[3] Schön G et al 1998 in Exploring the Quantum-Classical Frontier ed J R Friedman and S Han (Commack, NY: Nova Science) pp 405-440;

(Schön G et al 1998 Preprint cond-mat/9811029)

[4] Korotkov A N 1999 Phys. Rev. B 605737

[5] Gurvitz S A 1997 Phys. Rev. B 5615215

[6] Goan H-S et al 2001 Phys. Rev. B 63125326

[7] Devoret M H and Schoelkopf R J 2000 Nature 4061039

[8] Loss D and DiVincenzo D P 1998 Phys. Rev. A 57120

[9] Shnirman A et al 1997 Phys. Rev. Lett. 792371

[10] Milburn G J and Sun H B 1998 Contemporary Physics 39 number 1, pp 67-79

[11] Lindblad G 1976 Commun. Math. Phys. 48199

[12] Gardiner C W 1985 Handbook of Stochastic Methods for the Physical and Chemical Sciences (Berlin: Springer)

[13] Warszawski P et al 2002 Phys. Rev. A 65023802 
[14] Warszawski P and Wiseman H M 2003 J. Opt. B: Quantum Semiclass. Opt. 5 1-14; Warszawski P and Wiseman H M 2003 J. Opt. B: Quantum Semiclass. Opt. 5 15-28

[15] Sun H B and Milburn G J 1999 Phys. Rev. B 5910748

[16] de Jong M J M and Beenakker C W J 1997 in Mesoscopic Electron Transport ed L L Sohn et al NATO ASI Series E345 (Dordrecht: Kluwer) pp 225-258

[17] Gillespie D T 2000 J. Phys.: Condens. Matter 12 4195-4205

[18] Fano U 1946 Phys. Rev. 70 44; Fano U 1947 Phys. Rev. 7226

[19] Devoret M H and Grabert H 1991 Single Charge Tunnelling ed H Grabert and M H Devoret NATO ASI Series (New York: Plenum Press) pp 1-19 